Siberian Mathematical Journal, Vol. 54, No. 2, pp. 205-211, 2013

Original Russian Text Copyright (c) 2013 Astashkin S.V., Maligranda L., and Sukhanov R.S.

\title{
SEQUENCES OF INDEPENDENT WALSH FUNCTIONS IN BMO
}

\author{
(c) S. V. Astashkin, L. Maligranda, and R. S. Sukhanov
}

UDC 517.982.22:517.518.36

\begin{abstract}
Under examination are the sequences of independent Walsh functions in the space of functions of bounded mean oscillation. We study geometric properties of the subspaces spanned by the sequences; in particular, some necessary and sufficient conditions are found for such a subspace to be complemented.
\end{abstract}

DOI: $10.1134 /$ S0037446613020031

Keywords: Rademacher functions, Walsh functions, space of functions of bounded mean oscillation, complemented subspace

\section{Introduction and Statement of the Main Results}

The space $\mathrm{BMO}=\mathrm{BMO}[0,1]$ consists of the functions $f \in L_{1}[0,1]$ of bounded mean oscillation, that is, satisfying the condition

$$
\|f\|_{\mathrm{BMO}}:=\sup _{I} \frac{1}{|I|} \int_{I}\left|f(u)-f_{I}\right| d u<\infty,
$$

where the supremum is taken over all intervals $I \subset[0,1]$ and $f_{I}:=\frac{1}{|I|} \int_{I} f(u) d u$. If we use only the dyadic intervals $I_{m}^{i}=\left((i-1) 2^{-m}, i 2^{-m}\right]\left(m=0,1,2, \ldots, i=1, \ldots, 2^{m}\right)$ in the definition then we obtain the dyadic space $\mathrm{BMO}_{d}$. Clearly, $\mathrm{BMO} \subset \mathrm{BMO}_{d}$ and $\|f\|_{d}:=\|f\|_{\mathrm{BMO}_{d}} \leq\|f\|_{\mathrm{BMO}}$ for all $f \in$ BMO. Moreover, $L_{\infty}[0,1] \subset \mathrm{BMO}$ and $\|f\|_{\mathrm{BMO}} \leq\|f\|_{L_{\infty}}$ for all $f \in L_{\infty}=L_{\infty}[0,1]$. At the same time, $\mathrm{BMO} \neq L_{\infty}$ and $\mathrm{BMO}_{d} \neq \mathrm{BMO}$. For example, $\log |s-1 / 2| \chi_{[0,1]}(s) \in \mathrm{BMO} \backslash L_{\infty}$ and $\log |s-1 / 2| \chi_{[1 / 2,1]}(s) \in \mathrm{BMO}_{d} \backslash \mathrm{BMO}$.

Given an increasing sequence of positive integers $1 \leq p_{1}<p_{2}<\cdots$, consider the sequence of Walsh functions

$$
f_{k}=\prod_{i \in A_{k}} r_{i}, \quad A_{k} \subset\left\{p_{k}+1, p_{k}+2, \ldots, p_{k+1}\right\},
$$

where $r_{i}(t)$ are the Rademacher functions on [0,1]; i.e., $r_{i}(t)=\operatorname{sgn}\left[\sin \left(2^{i} \pi t\right)\right](i \in \mathbb{N})$.

In view of $[1$, Theorem 1$]$, the system $\left\{f_{k}\right\}_{k=1}^{\infty}$ defined by (1) is equivalent in $\mathrm{BMO}_{d}$ to the canonical basis of $l_{2}$; more exactly, for every finite sequence $a=\left(a_{k}\right)_{k=1}^{\infty}$, we have

$$
\frac{1}{\sqrt{2}}\|a\|_{2} \leq\left\|\sum_{k=1}^{\infty} a_{k} f_{k}\right\|_{d} \leq \sqrt{2}\|a\|_{2},
$$

where $\|a\|_{2}:=\left(\sum_{k=1}^{\infty} a_{k}^{2}\right)^{1 / 2}$. In this article it is proven that the subspace $\left[f_{k}\right]$ generated by a sequence $\left\{f_{k}\right\}_{k=1}^{\infty}$ (i.e. the closed linear span of $\left\{f_{k}\right\}$ ) is complemented in $\mathrm{BMO}_{d}$. Recall that the closed subspace $Y$ of a Banach space $X$ is called complemented in $X$ if there exists a bounded linear projection $P: X \rightarrow X$ whose range coincides with $Y$.

The main aim of the present article is the study of a system $\left\{f_{k}\right\}$ defined in (1) in the "usual" $\mathrm{BMO}$ space. It is important to note that the last space is invariant under translations in contrast to its

The first author was supported by the Russian Foundation for Basic Research (Grant 10-01-00077).

Samara and Luleå. Translated from Sibirskiu Matematicheskiu Zhurnal, Vol. 54, No. 2, pp. 270-278, March-April, 2013. Original article submitted January 19, 2012. 
dyadic version $\mathrm{BMO}_{d}$ and so it plays a more important role in analysis. We demonstrate that (2) may fail in BMO, while geometric properties of the subspace $\left[f_{k}\right]$ (in particular, its complementability) are essentially determined by evenness of the number of elements in the sets $A_{k}$. The present results are a natural continuation of those of the article [2] in which similar questions are studied for the Rademacher system. In particular, Theorems 1(a) and 3 can be considered as an extension of Theorems 2 and 4 in [2].

We present the main results of the article, where an expression of the form $f \asymp g$ means that $c f \leq g \leq C f$ for some constants $c>0$ and $C>0$ independent of all or some part of arguments of the norms (seminorms) $f$ and $g$. Moreover, we assume in what follows that $A_{k}$ and $f_{k}$ are defined by (1).

Theorem 1. (a) If every set $A_{k}$ contains an odd number of elements then, for some universal constants and every sequence $a=\left(a_{k}\right)_{k=1}^{\infty} \in l_{2}$, we have

$$
\left\|\sum_{k=1}^{\infty} a_{k} f_{k}\right\|_{\mathrm{BMO}} \asymp\|a\|_{2}+\sup _{s \geq 1}\left|\sum_{k=1}^{s} a_{k}\right| .
$$

(b) If every set $A_{k}$ contains an even number of elements then, for some universal constants and every sequence $a=\left(a_{k}\right)_{k=1}^{\infty} \in l_{2}$, we have

$$
\left\|\sum_{k=1}^{\infty} a_{k} f_{k}\right\|_{\mathrm{BMO}} \asymp\|a\|_{2}
$$

Theorem 2. If $\left\{k_{i}\right\}\left(k_{1}<k_{2}<\cdots\right)$ is the set of all $k \in \mathbb{N}$ for which the number of elements of all $A_{k}$ is odd then, for some universal constants and every sequence $a=\left(a_{k}\right)_{k=1}^{\infty} \in l_{2}$, we have

$$
\left\|\sum_{k=1}^{\infty} a_{k} f_{k}\right\|_{\mathrm{BMO}} \asymp\|a\|_{2}+\sup _{s \geq 1}\left|\sum_{i=1}^{s} a_{k_{i}}\right| .
$$

Recall that a sequence $\left\{x_{n}\right\}_{n=1}^{\infty}$ of elements in a Banach space $X$ is called a basic sequence if it is a basis of the closed linear span $\left[x_{n}\right]$. Moreover, a basic sequence $\left\{x_{n}\right\}_{n=1}^{\infty}$ is referred to as unconditional whenever, for every permutation $\pi$ of positive integers, the sequence $\left\{x_{\pi(k)}\right\}_{k=1}^{\infty}$ is a basic sequence in $X$ as well. As is known (see, for instance, [3, Theorem 1.1]), a basic sequence $\left\{x_{n}\right\}_{n=1}^{\infty}$ is unconditional in $X$ if and only if the convergence of a series $\sum_{n=1}^{\infty} a_{n} x_{n}\left(a_{n} \in \mathbb{R}\right)$ ensures the convergence of a series $\sum_{n=1}^{\infty} \theta_{n} a_{n} x_{n}$, where $\left\{\theta_{n}\right\}_{n=1}^{\infty}$ is an arbitrary sequence of signs, i.e., $\theta_{n}= \pm 1$. Thereby, Theorem 2 yields

Corollary 1. The following are equivalent:

(1) $\left\{f_{k}\right\}_{k=1}^{\infty}$ is an unconditional basic sequence in BMO;

(2) $\left\{f_{k}\right\}_{k=1}^{\infty}$ is equivalent in BMO to the canonical basis of $l_{2}$;

(3) all sets $A_{k}$, except possibly finitely many, contain an even number of elements.

The last result gives a necessary and sufficient condition for the subspace $\left[f_{k}\right]$ to be complemented in BMO.

Theorem 3. The following are equivalent:

(1) the subspace $\left[f_{k}\right]$ generated by $\left\{f_{k}\right\}_{k=1}^{\infty}$ is complemented in BMO;

(2) all sets $A_{k}$, except possibly finitely many, contain an even number of elements.

\section{Proofs}

To prove Theorem 1, we need two auxiliary propositions; the former of them is the contents of Problem 12(b) in [4, p. 266] (see its proof with the constants below in [2, Proposition 1]).

Define the functional $A(f)=\sup _{I_{1}, I_{2}}\left|f_{I_{1}}-f_{I_{2}}\right|$, where $I_{1}$ and $I_{2}$ are adjacent dyadic intervals of the same length. 
Proposition 1. For all $f \in L_{1}[0,1]$, we have

$$
\frac{1}{3}\left(\|f\|_{d}+A(f)\right) \leq\|f\|_{\mathrm{BMO}} \leq 32\left(\|f\|_{d}+A(f)\right) .
$$

Proposition 2. Let functions $f_{k}$ be defined in (1) and let a sequence of reals $a=\left(a_{k}\right)$ be finite. Put $f:=\sum_{k \geq 1} a_{k} f_{k}$.

(i) If every set $A_{k}$ contains an odd number of elements, then

$$
\frac{2}{3} \max _{s \geq 1}\left|\sum_{k=1}^{s} a_{k}\right| \leq A(f) \leq 8 \max _{s \geq 1}\left|\sum_{k=1}^{s} a_{k}\right| .
$$

(ii) If every set $A_{k}$ contains an even number of elements, then

$$
A(f)=2 \max _{s \geq 1}\left|a_{s}\right| .
$$

Proof. Let $I$ be an arbitrary dyadic interval of length $2^{-r}$, i.e., $I=I_{r}^{i}=\left((i-1) 2^{-r}, i 2^{-r}\right]$. Put $m_{k}:=\min A_{k}, M_{k}:=\max A_{k}$, and $s=s(r)=\max \left\{k: M_{k} \leq r\right\}$. If $M_{k}>r$ for all $k$ then we set $s=0$. In this case

$$
\left(f_{k}\right)_{I}= \begin{cases}\operatorname{sgn}\left(\left.f_{k}\right|_{I}\right), & k \leq s \\ 0, & k>s\end{cases}
$$

Indeed, if $k \leq s$ then $f_{k}$ is constant on $I$. In the case of $k>s$, we have $f_{k}=\prod_{i \in B_{k} \cup C_{k}} r_{i}$, and $r_{i}$ are constant on $I$ for all $i \in B_{k}$, while $\int_{I} \prod_{i \in C_{k}} r_{i}=0$. Hence, since $r_{i}, i \in C_{k}$, are independent with respect to $I$, we infer

$$
\left(f_{k}\right)_{I}=\frac{1}{|I|} \int_{I} f_{k}(t) d t=\frac{c}{|I|} \int_{I} \prod_{i \in C_{k}} r_{i}(t) d t=\frac{c}{|I|} \prod_{i \in C_{k}} \int_{I} r_{i}(t) d t=0,
$$

where $c$ is the value of $\prod_{i \in B_{k}} r_{i}$ on $I$.

Therefore, if $I_{1}$ and $I_{2}$ are adjacent dyadic intervals of length $2^{-r}$ then

$$
f_{I_{1}}-f_{I_{2}}=\sum_{k=1}^{s} a_{k} \operatorname{sgn}\left(\left.f_{k}\right|_{I_{1}}\right)-\sum_{k=1}^{s} a_{k} \operatorname{sgn}\left(\left.f_{k}\right|_{I_{2}}\right)=\sum_{k=1}^{s} a_{k}\left[\operatorname{sgn}\left(\left.f_{k}\right|_{I_{1}}\right)-\operatorname{sgn}\left(f_{k} \mid I_{2}\right)\right] .
$$

Let $I$ be the least dyadic interval containing the union of $I_{1}$ and $I_{2}$. Assume that its length is $2^{-j}$. The definition of $I$ implies that the union $I_{1} \cup I_{2}$ lies in the middle of $I$ (let $I_{1}$ lie on the left). As is easily seen, the index $j$ runs over all integers from 0 to $r-1$ in dependence on $I_{1}$ and $I_{2}$.

Below, we need the following notation: If $a, b \in \mathbb{N}$ and $a \leq b$ then $[a, b]:=\{i \in \mathbb{N}: a \leq i \leq b\}$. In view of the definition of $s$, we have $\bigcup_{k=1}^{s} A_{k} \subset[1, r]$. Put

$$
B:=\bigcup_{l=1}^{s}\left[m_{k}, M_{k}\right] \quad \text { and } \quad B^{\prime}:=[1, r] \backslash B=\bigcup_{l=1}^{s+1}\left[M_{l-1}+1, m_{l}-1\right],
$$

where $M_{0}=0$ and $m_{s+1}=r+1$. Moreover, in what follows, $|A|$ stands for the number of elements in a set $A \subset \mathbb{N}$.

Let us compare the values of $f_{k}, 1 \leq k \leq s$, on the intervals $I_{1}$ and $I_{2}$. If $m_{k}>j+1$, then all $r_{i}$ with indices from $A_{k}$ change their signs under the passage from $I_{1}$ to $I_{2}$; in this case $\left.r_{i}\right|_{I_{1}}=-1$ and $\left.r_{i}\right|_{I_{2}}=1$. Hence, for these $k$, we have $\left.f_{k}\right|_{I_{1}}=(-1)^{\left|A_{k}\right|}$ and $\left.f_{k}\right|_{I_{2}}=1$. If $M_{k} \leq j$ then all $r_{i}$ with indices from $A_{k}$ are constant on $I$ and so $\left.f_{k}\right|_{I_{1}}=\left.f_{k}\right|_{I_{2}}$. In dependence on which set $B$ or $B^{\prime}$ contains the number $j+1$, we examine two cases. 
(a) $j+1 \in B^{\prime}$. In other words, $M_{l-1}+1 \leq j+1 \leq m_{l}-1$ for some $l=1,2, \ldots, s+1$. If $l=s+1$ then $j \geq M_{s}$ and so from (9) and the above remarks it follows that $f_{I_{1}}-f_{I_{2}}=0$. In the case when the previous inequality is fulfilled for $l=1,2, \ldots, s$, we see that $M_{l-1} \leq j<m_{l}-2$ and, by the same reasons,

$$
f_{I_{1}}-f_{I_{2}}=\sum_{k=l}^{s} a_{k}\left(\left.f_{k}\right|_{I_{1}}-\left.f_{k}\right|_{I_{2}}\right)=\sum_{k=l}^{s} a_{k}\left((-1)^{\left|A_{k}\right|}-1\right) .
$$

Since $j$ takes all values from 0 to $r-1$, equation (10) is true for $l \in \mathscr{F}_{1}$, where the set $\mathscr{F}_{1}$ consists of those numbers $k$ for which the segment $\left[M_{k-1}+1, m_{k}-1\right]$ is nonempty (if $2 \leq k \leq s$, then the latter is equivalent to the fact that there is a "gap" between the sets $A_{k-1}$ and $A_{k} ; 1 \in \mathscr{F}_{1}$ if $1 \notin A_{1}$ ).

(b) $j+1 \in B$. In this case $m_{l} \leq j+1 \leq M_{l}$ for some $l=1,2, \ldots, s$ and the functions $r_{i}$ change their signs under the passage from $I_{1}$ to $I_{2}$ if $i \in A_{l}^{(j)}:=\left\{p \in A_{l}: p>j\right\}$ and they are constant on $I$ if $i \in A_{l} \backslash A_{l}^{(j)}$. Hence, $\left.f_{l}\right|_{I_{2}}=\left.(-1)^{\left|A_{l}^{(j)}\right|} f_{l}\right|_{I_{1}}$. Since $j+1<m_{l+1}$ and $j \geq M_{l-1}$, taking into account the above remarks, by (9), we establish

$$
f_{I_{1}}-f_{I_{2}}=\left.a_{l} f_{l}\right|_{I_{1}}\left(1-(-1)^{\left|A_{l}^{(j)}\right|}\right)+\sum_{k=l+1}^{s} a_{k}\left((-1)^{\left|A_{k}\right|}-1\right) .
$$

Assume that all numbers $\left|A_{k}\right|$ are odd. First of all, (10) implies that

$$
\left|f_{I_{1}}-f_{I_{2}}\right|=2\left|\sum_{k=l}^{s} a_{k}\right|, \quad l \in \mathscr{F}_{1} .
$$

If there exists $j$ such that $m_{l} \leq j+1 \leq M_{l}$ and either $\left.f_{l}\right|_{I_{1}}=-1$ or $\left|A_{l}^{(j)}\right|$ is even, then (11) ensures (12) (in the second case $l$ should be replaced with $l+1$ ). Denote by $\mathscr{F}$ the set of $l$ such that (12) holds. Clearly, $\mathscr{F} \supset \mathscr{F}_{1}$.

It is easy to see that, for $l=1,2, \ldots, s$, we have one more possibility. If $j=m_{l}-1$ then $A_{l}^{(j)}=A_{l}$. Since $\left.r_{m_{l}}\right|_{I_{1}}=1$ and $\left.r_{i}\right|_{I_{1}}=-1$, if $i \in A_{l}, i \neq m_{l}$ then $\left.f_{l}\right|_{I_{1}}=1$. Hence, (11) yields

$$
\left|f_{I_{1}}-f_{I_{2}}\right|=2\left|\sum_{k=l+1}^{s} a_{k}-a_{l}\right|, \quad l=1,2, \ldots, s .
$$

For a given pair of adjacent dyadic intervals $I_{1}$ and $I_{2}$, the quantity $\left|f_{I_{1}}-f_{I_{2}}\right|$ is determined by equalities (12) or (13). Therefore, according to the definition of the functional $A(f)$ we obtain

$$
A(f)=2 \max \left(\max _{1 \leq l \leq s<\infty}\left|\sum_{k=l+1}^{s} a_{k}-a_{l}\right|, \max _{\substack{1 \leq l \leq s<\infty \\ l \in \mathscr{F}}}\left|\sum_{k=l}^{s} a_{k}\right|\right) .
$$

Moreover, every finite sequence $\left(a_{k}\right)$ satisfies the inequality

$$
\frac{1}{3} \max _{1 \leq l \leq s<\infty}\left|\sum_{k=l}^{s} a_{k}\right| \leq \max _{1 \leq l \leq s<\infty}\left|\sum_{k=l+1}^{s} a_{k}-a_{l}\right| \leq 2 \max _{1 \leq l \leq s<\infty}\left|\sum_{k=l}^{s} a_{k}\right| .
$$

Indeed, on the one hand, for arbitrary $1 \leq l \leq s$, we have

$$
\left|\sum_{k=l}^{s} a_{k}\right| \leq\left|\sum_{k=l+1}^{s} a_{k}-a_{l}\right|+2\left|a_{l}\right| \leq 3 \max _{1 \leq l \leq s<\infty}\left|\sum_{k=l+1}^{s} a_{k}-a_{l}\right|
$$

(letting in the case $s=l$ the sum $\sum_{k=l+1}^{s} a_{k}$ to be equal to zero). Conversely, for $1 \leq l \leq s$, we infer

$$
\left|\sum_{k=l+1}^{s} a_{k}-a_{l}\right| \leq\left|\sum_{k=l+1}^{s} a_{k}\right|+\left|a_{l}\right| \leq 2 \max _{1 \leq l \leq s<\infty}\left|\sum_{k=l}^{s} a_{k}\right| .
$$


Moreover,

$$
\max _{s \geq 1}\left|\sum_{k=1}^{s} a_{k}\right| \leq \max _{1 \leq l \leq s<\infty}\left|\sum_{k=l}^{s} a_{k}\right| \leq 2 \max _{s \geq 1}\left|\sum_{k=1}^{s} a_{k}\right| .
$$

The left inequality in (15) is obvious and the right one follows from the estimate

$$
\left|\sum_{k=l}^{s} a_{k}\right| \leq\left|\sum_{k=1}^{s} a_{k}-\sum_{k=1}^{l} a_{k}\right| \leq\left|\sum_{k=1}^{s} a_{k}\right|+\left|\sum_{k=1}^{l} a_{k}\right| \leq 2 \max _{s \geq 1}\left|\sum_{k=1}^{s} a_{k}\right|
$$

which is valid for arbitrary $1 \leq l \leq s$. As a result, the left inequality in (7) follows from the above inequality for $A(f)$ and the left inequality in (14). To obtain the right inequality in (7), it suffices to use the right-hand sides in (14) and (15).

The case when every set $A_{k}$ contains an even number of elements is much simpler. In fact, equalities (10) and (11) ensure that either $f_{I_{1}}-f_{I_{2}}=0$ or $\left|f_{I_{1}}-f_{I_{2}}\right|=2\left|a_{l}\right|, l=1,2, \ldots, s$. Thereby, (8) is proved.

Proof of Theorem 1. It suffices to apply Propositions 1 and 2 together with (2).

Proof of Theorem 2. Let $f:=\sum_{k=1}^{\infty} a_{k} f_{k}$. Relations (10) and (11) and the same arguments as in the proof of Proposition 2 imply that

$$
A(f) \asymp \max \left(\sup _{k=1,2, \ldots}\left|a_{k}\right|, \sup _{s \geq 1}\left|\sum_{i=1}^{s} a_{k_{i}}\right|\right),
$$

where $\left\{k_{i}\right\}\left(k_{1}<k_{2}<\cdots\right)$ is the set of all $k \in \mathbb{N}$ such that $\left|A_{k}\right|$ is odd. Thereby, Proposition 1 and (2) justify the claim.

To prove Theorem 3, we need one more auxiliary statement about the block bases of a sequence $\left\{f_{k}\right\}_{k=1}^{\infty}$ in BMO.

Denote by $U=\left\{u_{n}\right\}_{n=1}^{\infty}$ an arbitrary block basis of the sequence $\left\{f_{k}\right\}_{k=1}^{\infty}$, i.e.,

$$
u_{n}=\sum_{k=s_{n}+1}^{s_{n+1}} a_{k} f_{k}, \quad n=1,2, \ldots
$$

where $1 \leq s_{1}<s_{2}<\cdots$ and $a_{k} \in \mathbb{R}$. Moreover, let

$$
\gamma_{n}(U)=\sum_{k=s_{n}+1}^{s_{n+1}} a_{k}, \quad n=1,2, \ldots
$$

Proposition 3. If a sequence $\left\{f_{k}\right\}_{k=1}^{\infty}$ contains infinitely many elements with an odd $\left|A_{k}\right|$, then the closed linear span $\left[f_{k}\right] \subset \mathrm{BMO}$ contains a subspace $E$ isomorphic to $c_{0}$ and complemented in $\left[f_{k}\right]$.

Proof. Let $\mathscr{E}$ be the set of all $k \in \mathbb{N}$ for which $\left|A_{k}\right|$ is odd. By condition, $|\mathscr{E}|=\infty$. Propositions 1 and 2 together with (2) ensure the existence of a block basis $U=\left\{u_{n}\right\}_{n=1}^{\infty}$ satisfying the conditions

(a) $\left\|u_{n}\right\|_{\mathrm{BMO}}=1, n=1,2, \ldots$;

(b) $\left\|u_{n}\right\|_{d} \leq \sqrt{2}\left(\sum_{k=s_{n}+1}^{s_{n+1}} a_{k}^{2}\right)^{1 / 2} \leq 2^{-n-5}, n=1,2, \ldots$;

(c) $\gamma_{n}(U)=0, n=1,2, \ldots$;

(d) $\left\{k: a_{k} \neq 0\right\} \subset \mathscr{E}$.

Indeed, assume that we already constructed $u_{1}, \ldots, u_{n-1}$. Given $n \in \mathbb{N}$, there exist a sufficiently large $m_{n} \in \mathbb{N}$ and numbers $b_{k} \geq 0, k=1,2, \ldots, m_{n}$ such that

$$
\left(\sum_{k=1}^{m_{n}} b_{k}^{2}\right)^{1 / 2} \leq \frac{2}{9} \cdot 2^{-n-7} \quad \text { and } \quad \sum_{k=1}^{m_{n}} b_{k}=1
$$


Put $a_{k}^{\prime}=b_{k}$ if $k=1,2, \ldots, m_{n}$ and $a_{k}^{\prime}=-b_{k-m_{n}}$, if $k=m_{n}+1, \ldots, 2 m_{n}$. Choose a sufficiently large number $s_{n+1}>s_{n}$ such that the segment $\left\{s_{n}+1, \ldots, s_{n+1}\right\}$ of positive integers contains at least $2 m_{n}$ elements of $\mathscr{E}$, and introduce new indices for $a_{k}^{\prime}$ from the intersection $\left\{s_{n}+1, \ldots, s_{n+1}\right\} \cap \mathscr{E}$ preserving the order. For the remaining numbers $k$ from the set $\left\{s_{n}+1, \ldots, s_{n+1}\right\}$, we put $a_{k}^{\prime}=0$. If $u_{n}^{\prime}:=\sum_{k=s_{n}+1}^{s_{n+1}} a_{k}^{\prime} f_{k}$, then, in view of (2) and the first relation in (16), we find that

$$
\left\|u_{n}^{\prime}\right\|_{d} \leq \sqrt{2} \cdot 2\left(\sum_{k=1}^{m_{n}} b_{k}^{2}\right)^{1 / 2} \leq \frac{2}{9} \cdot 2^{-n-5} .
$$

Moreover, since the definition of $a_{k}^{\prime}$ and the second equality in (16) imply that

$$
\max _{s_{n}+1 \leq s \leq s_{n+1}}\left|\sum_{k=s_{n}+1}^{s} a_{k}^{\prime}\right|=\sum_{k=1}^{m_{n}} b_{k}=1,
$$

Propositions 1 and 2 yield

$$
\frac{2}{3} \leq A\left(u_{n}^{\prime}\right) \leq 8 \quad \text { and } \quad \frac{2}{9} \leq\left\|u_{n}^{\prime}\right\|_{\mathrm{BMO}} \leq 288 .
$$

Thereby, it is easy to see that the function $u_{n}:=\frac{u_{n}^{\prime}}{\left\|u_{n}^{\prime}\right\|_{\text {BMO }}}$ meets (a), (b), and (d). Condition (c) holds since $\sum_{k=s_{n}+1}^{s_{n+1}} a_{k}^{\prime}=0$ by construction.

Let us show that the subspace $E:=\left[u_{n}\right]$, spanned by the functions of this block basis in BMO, is isomorphic to $c_{0}$.

Take $f \in\left[u_{n}\right]$. If $f=\sum_{n=1}^{\infty} \beta_{n} u_{n}\left(\beta_{n} \in \mathbb{R}\right)$ then

$$
f=\sum_{n=1}^{\infty}\left(\sum_{k=s_{n}+1}^{s_{n+1}} \beta_{n} a_{k} f_{k}\right)=\sum_{k=1}^{\infty} \gamma_{k} f_{k},
$$

where $\gamma_{k}=\beta_{n} a_{k}$ for $k=s_{n}+1, \ldots, s_{n+1}$. Assuming that $p, q \in \mathbb{N}$ satisfy the inequalities $s_{n-1} \leq p<$ $s_{n}<s_{n+l}<q \leq s_{n+l+1}$ with some positive integers $n$ and $l$, we estimate the sum $\sum_{k=p}^{q} \gamma_{k}$. In view of (d), (c), and (a), together with Propositions 1 and 2(i), we infer

$$
\begin{gathered}
\left|\sum_{k=p}^{q} \gamma_{k}\right|=\left|\sum_{k=p}^{s_{n}} \gamma_{k}+\sum_{k=s_{n}+1}^{s_{n+l}} \gamma_{k}+\sum_{k=s_{n+l}+1}^{q} \gamma_{k}\right| \\
=\left|\sum_{k=p}^{s_{n}} \beta_{n-1} a_{k}+\sum_{i=n}^{n+l-1} \sum_{k=s_{i}+1}^{s_{i+1}} \beta_{i} a_{k}+\sum_{k=s_{n+l}+1}^{q} \beta_{n+l} a_{k}\right| \\
\leq\left|\beta_{n-1}\right|\left|\sum_{k=p}^{s_{n}} a_{k}\right|+\sum_{i=n}^{n+l-1}\left|\beta_{i}\right|\left|\sum_{k=s_{i}+1}^{s_{i+1}} a_{k}\right|+\left|\beta_{n+l}\right|\left|\sum_{k=s_{n+l}+1}^{q} a_{k}\right| \\
\leq \sup _{n}\left|\beta_{n}\right|\left(\left|\sum_{k=p}^{s_{n}} a_{k}\right|+\left|\sum_{k=s_{n+l}+1}^{q} a_{k}\right|\right) \leq \sup _{n}\left|\beta_{n}\right|\left(\frac{3}{2} A\left(u_{n-1}\right)+\frac{3}{2} A\left(u_{n+l}\right)\right) \\
\leq \frac{9}{2}\left(\left\|u_{n-1}\right\|_{\mathrm{BMO}}+\left\|u_{n+l}\right\|_{\mathrm{BMO}}\right)\left\|\left\{\beta_{n}\right\}\right\|_{c_{0}}=9\left\|\left\{\beta_{n}\right\}\right\|_{c_{0}} .
\end{gathered}
$$

Applying Theorem 1(a), inequality (2), and properties (a) and (b), we find that

$$
\begin{aligned}
\|f\|_{\mathrm{BMO}} \leq & C\left(\|f\|_{d}+\sup _{q \geq 1}\left|\sum_{k=1}^{q} \gamma_{k}\right|\right) \leq C\left(\left\|\sum_{n=1}^{\infty} \beta_{n} u_{n}\right\|_{d}+9\left\|\left\{\beta_{n}\right\}\right\|_{c_{0}}\right) \\
\leq & C\left(\sum_{n=1}^{\infty} 2^{-n}+9\right)\left\|\left\{\beta_{n}\right\}\right\|_{c_{0}}=10 C\left\|\left\{\beta_{n}\right\}\right\|_{c_{0}} .
\end{aligned}
$$


On the other hand, Proposition 1, (a), and (b) imply that

$$
A\left(u_{n}\right) \geq \frac{1}{32}\left\|u_{n}\right\|_{\mathrm{BMO}}-\left\|u_{n}\right\|_{d} \geq \frac{1}{32}-2^{-n-5} \geq \frac{1}{64}
$$

for all $n \in \mathbb{N}$. Hence, applying Propositions 1 and 2 once again (also see inequalities (7) and (15)), we arrive at the inequality

$$
\|f\|_{\mathrm{BMO}} \geq \frac{1}{3} A(f) \geq \frac{1}{9} \sup _{n \in \mathbb{N}} \sup _{s_{n}+1 \leq p<q \leq s_{n+1}}\left|\sum_{k=p}^{q} \beta_{n} a_{k}\right| \geq \frac{1}{72} A\left(u_{n}\right)\left\|\left\{\beta_{n}\right\}\right\|_{c_{0}} \geq \frac{1}{72 \cdot 64}\left\|\left\{\beta_{n}\right\}\right\|_{c_{0}} .
$$

Thus, the system $\left\{u_{n}\right\}$ is equivalent in BMO to the canonical basis of $c_{0}$, and so $E$ is isomorphic to $c_{0}$. Since $\left[f_{k}\right]$ is separable, by the Sobczyk theorem (see, for instance, [5, Corollary 2.5.9]), $E$ is complemented in $\left[f_{k}\right]_{k=1}^{\infty}$ and the proposition is proved.

Proof of Theorem 3. First, assume that all sets $A_{k}$, except possibly finitely many, contain an even number of elements. By Corollary $1,\left\{f_{k}\right\}_{k=1}^{\infty}$ is equivalent in BMO to the canonical basis of $l_{2}$. On the other hand, one of the consequences of the classical John-Nirenberg inequality (see, for instance, $\left[6\right.$, Remark 3.19]) is the embedding BMO $\subset L_{p}[0,1]$ for $1 \leq p<\infty$. Since $\left\{f_{k}\right\}_{k=1}^{\infty}$ is an orthonormal system in $L_{2}[0,1]$, the corresponding orthogonal projection $P$ is bounded in $L_{2}[0,1]$, its range is the subspace $\left[f_{k}\right]$, and the sequence $\left\{f_{k}\right\}_{k=1}^{\infty}$ in $L_{2}[0,1]$ is also equivalent to the canonical basis of $l_{2}$. Hence, for every function $f \in \mathrm{BMO}$, we have

$$
\|P f\|_{\mathrm{BMO}} \asymp\|P f\|_{L_{2}} \leq\|P\|\|f\|_{L_{2}} \leq C\|P\|\|f\|_{\mathrm{BMO}}
$$

i.e., $P$ is bounded in BMO and its range coincides with the closed linear span $\left[f_{k}\right]$ in this space. Thereby, the subspace $\left[f_{k}\right]$ is complemented in BMO.

We prove the converse. Assume that $\left[f_{k}\right]$ is complemented in BMO and the set $\mathscr{E}$ of the numbers $k \in \mathbb{N}$ with an odd $\left|A_{k}\right|$ is infinite. Let $P_{1}:$ BMO $\rightarrow\left[f_{k}\right]$ be a bounded linear projection whose range coincides with $\left[f_{k}\right]$. By Proposition 3 , there exists a subspace $E$ complemented in $\left[f_{k}\right]$ and isomorphic to $c_{0}$. Let $P_{2}:\left[f_{k}\right] \rightarrow E$ be a bounded linear projection whose range is $E$. In this case $P:=P_{2} \circ P_{1}$ is a linear projection bounded in $\mathrm{BMO}$ with the range $E$. Hence, BMO contains a complemented subspace isomorphic to $c_{0}$. Since BMO is a dual space (more exactly, BMO $=\left(\operatorname{Re} H_{1}\right)^{*}$; see, for instance, $[3$, Theorem 5.5]), it contradicts to the known Bessaga-Pełczyński result that a dual space does not contain a complemented subspace isomorphic to $c_{0}$ (see [7, Corollary 4]). Thus, if the set $\mathscr{E}$ of numbers $k \in \mathbb{N}$ with an odd $\left|A_{k}\right|$ is infinite, the subspace $\left[f_{k}\right]$ is not complemented in BMO, and the theorem is proved.

\section{References}

1. Müller P. F. and Schechtman G., "On complemented subspaces of $H^{1}$ and VMO," Lecture Notes Math., 1376, 113-125 (1989).

2. Astashkin S. V., Leibov M., and Maligranda L., "Rademacher functions in BMO," Studia Math., 205, No. 1, 83-100 (2011).

3. Kashin B. S. and Saakyan A. A., Orthogonal Series, Amer. Math. Soc., Providence (1989).

4. Garnett J. B., Bounded Analytic Functions, Springer-Verlag, New York (2007).

5. Albiac F. and Kalton N. J., Topics in Banach Space Theory (Graduated Texts in Mathematics; V. 233), Springer-Verlag, New York (2006)

6. Korenovskii A., Mean Oscillations and Equimeasurable Rearrangements of Functions, Springer-Verlag, Berlin and Heidelberg (2007).

7. Bessaga C. and Pełczyński A., "Some remarks on conjugate spaces containing subspaces isomorphic to the space $c_{0}$," Bull. Acad. Polon. Sci. Sér. Sci. Math. Astronom. Phys., 6, 249-250 (1958).

S. V. Astashinin; R. S. Sukhanov

Samara State University, Samara, Russia

E-mail address: astash@samsu.ru; surose@yandex.ru

L. MALIGRANDA

Lule University of Technology, Lule , SWeden

E-mail address: lech.maligranda@ltu.se 